\title{
Automatic vegetation identification in Google Earth images using a convolutional neural network: A case study for Japanese bamboo \\ forests
}

Shuntaro Watanabe ${ }^{1}$, Kazuaki Sumi ${ }^{2}$, Takeshi Ise ${ }^{1,3}$

1. Field Science Education and Research Center (FSERC), Kyoto University, Kitashirakawaoiwake-cho, Sakyo-ku, Kyoto 606-8502, Japan

2. Graduate School of Agriculture, Kyoto University, Kitashirakawaoiwake-cho, Sakyo-ku, Kyoto 606-8502, Japan

3. PRESTO, Japan Science and Technology Agency, 7 Goban-cho, Chiyoda-ku, Tokyo 102-0076, Japan

Running head: Vegetation identification using deep learning

Author for correspondence: SW

E-mail: sh.watanabe1006@gmail.com (SW) 


\section{ABSTRACT}

2 Classifying and mapping vegetation are very important tasks in environmental science

3 and natural resource management. However, these tasks are not easy because

4 conventional methods such as field surveys are highly labor intensive. Automatic

5 identification of target objects from visual data is one of the most promising ways to

6 reduce the costs for vegetation mapping. Although deep learning has become a new

7 solution for image recognition and classification recently, in general, detection of

8 ambiguous objects such as vegetation still is considered difficult. In this paper, we

9 investigated the potential for adapting the chopped picture method, a recently described

10 protocol for deep learning, to detect plant communities in Google Earth images. We

11 selected bamboo forests as the target. We obtained Google Earth images from three

12 regions in Japan. By applying the deep convolutional neural network, the model

13 successfully learned the features of bamboo forests in Google Earth images, and the

14 best trained model correctly detected $97 \%$ of the targets. Our results show that

15 identification accuracy strongly depends on the image resolution and the quality of

16 training data. Our results also highlight that deep learning and the chopped picture

17 method can potentially become a powerful tool for high accuracy automated detection

18 and mapping of vegetation.

20 Key Words

21 Deep learning, Convolutional neural network, Vegetation mapping, Google Earth

22 imagery 


\section{INTRODUCTION}

25 Classifying and mapping vegetation are essential tasks for environmental science research and natural resource management ${ }^{1}$. Traditional methods (e.g., field surveys,

27 literature reviews, manual interpretation of aerial photographs), however, are not effective for acquiring vegetation data because they are labor intensive and often economically expensive. The technology of remote sensing offers a practical and economical means to acquire information on vegetation cover, especially over large $\operatorname{areas}^{2}$. Because of its systematic observations at various scales, remote sensing technology potentially can enable classification and mapping of vegetation at high temporal resolutions.

Detection of discriminating visual features is one of the most important steps in almost any computer vision problem, including in the field of remote sensing. Since conventional methods such as support vector machines ${ }^{3}$ require hand-designed, timeconsuming feature extraction, substantial efforts have been dedicated to development of methods for the automatic extraction of features. Recently, deep learning has become a new solution for image recognition and classification because this new method does not require the manual extraction of features. inspired by the structure and function of the brain called artificial neural networks. Deep learning involves the learning of features and classifiers simultaneously, and it uses

44 training data to categorize image content without a priori specification of image

45 features. Among all deep learning-based networks, the convolutional neural network $(\mathrm{CNN})$ is the most popular for learning visual features in computer vision applications

47 including remote sensing. Recent research has shown that $\mathrm{CNN}$ is effective for diverse 
applications $^{4-7}$. Given its success, deep learning has been used intensively in several distinct tasks for different academic and industrial fields including plant science. Recent research has shown that the deep learning technique can successfully detect plant disease, correctly classify the plant specimens in a herbarium ${ }^{8-10}$

Deep learning is a promising technology also in the field of remote sensing ${ }^{11,12}$. Recently, Guirado et al., (2017) ${ }^{13}$ demonstrated that the deep learning technique successfully detect plant species of conservation concern and it provides better results than the conventional object detection methods. However, application of deep learning to vegetation mapping are not sufficient yet because vegetation in the aerial image often shows ambiguous and amorphous shape, and automatic object identification including deep learning tends not to work well on such objects.

Recently, Ise et al., (2018) ${ }^{14}$ developed a method to extract relevant characteristics from ambiguous and amorphous objects. This method dissects the images into numerous small squares and efficiently produces the training images. By using this method, Ise et al. $(2018)^{14}$ correctly classified three moss species and "nonmoss" objects in test images with an accuracy of more than $90 \%$.

In this paper, we investigated the potential for adapting a deep learning model and the chopped picture method to automatic vegetation detection in Google Earth images, and bamboo forests were used as the target. In recent years, bamboo has become invasive in Japan. The bamboo species moso (Phyllostachys edulis) and madake (P. bambusoides Siebold) are the two main types of exotic bamboo. Since the 1970s, the bamboo industry in Japan had declined as a result of cheaper bamboo imports and heavy labor costs ${ }^{15}$. Consequently, many bamboo plantations were left unmanaged, which led to the eventual invasion of adjacent native vegetation ${ }^{16-18}$. 
In this study, we specifically addressed the following questions: 1) how does the

73 resolution of images affect the accuracy of detection; 2) how does the chopping size of

74 training images affect the accuracy of detection; and 3) can a model that learned in one

75 geographical location work well for a different location?

76

77 MATERIALS AND METHODS

78 Target area and image acquisition

79 In this study, we chose three regions (Sanyo-Onoda, Ide, and Isumi) in Japan to conduct

80 the analyses (Figure 1). We used Google Earth as the source of imagery. From a given

81 sampling location, we obtained the images at zoom levels of $1 / 500(\sim 0.13 \mathrm{~m} / \mathrm{pixel}$

82 spatial resolution), $1 / 1000(\sim 0.26 \mathrm{~m} /$ pixel spatial resolution), and $1 / 2500(\sim 0.65 \mathrm{~m} /$ pixel

83 spatial resolution). 


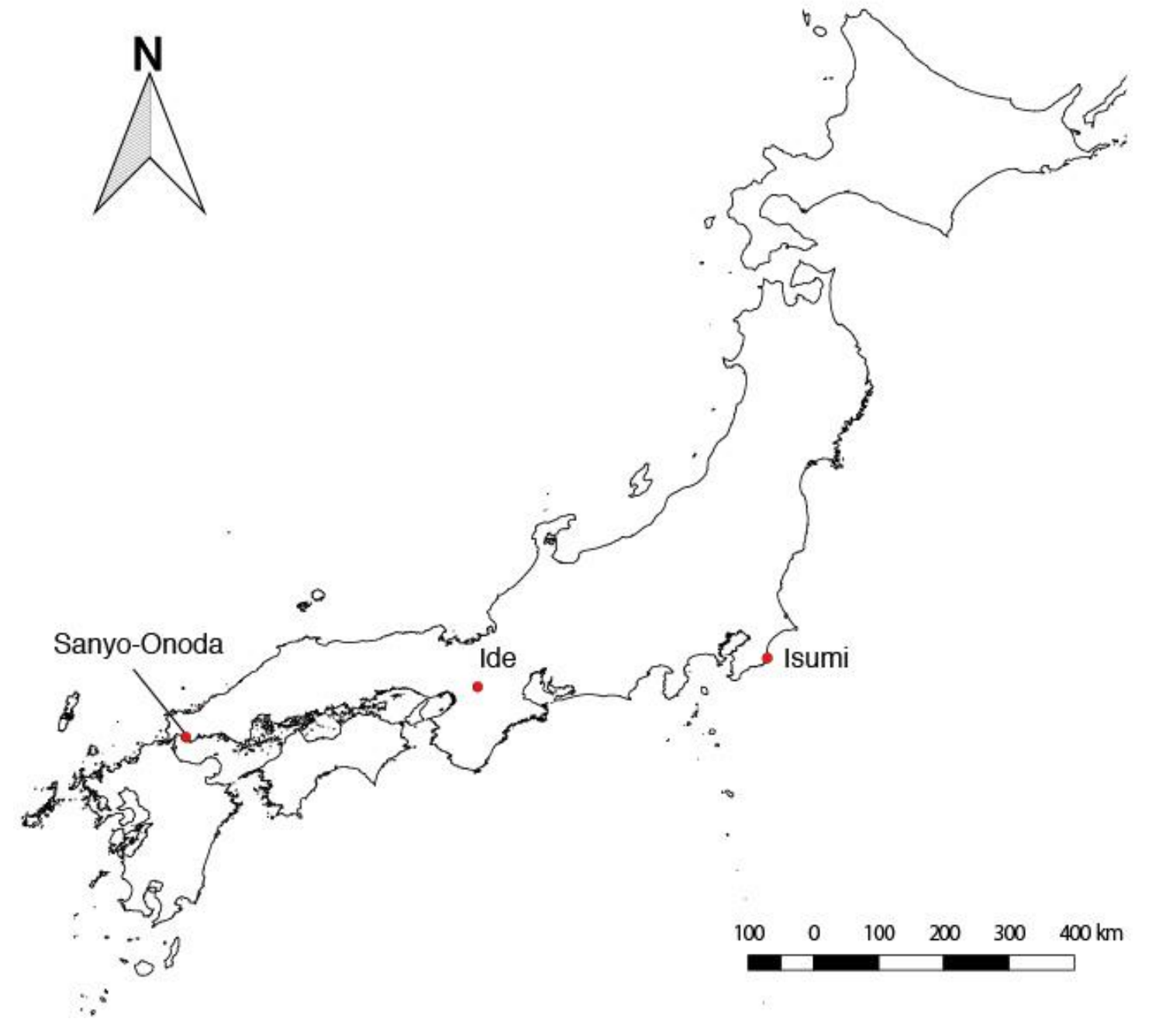

85 FIGURE 1 Target regions of this research.

86

\section{Methods and background concepts for the neural networks}

88 In this study, we employed convolutional neural networks (CNN; Figure 2). A CNN is a

89 special type of feedforward neural network that consists of a convolutional layer and 
pooling layer.

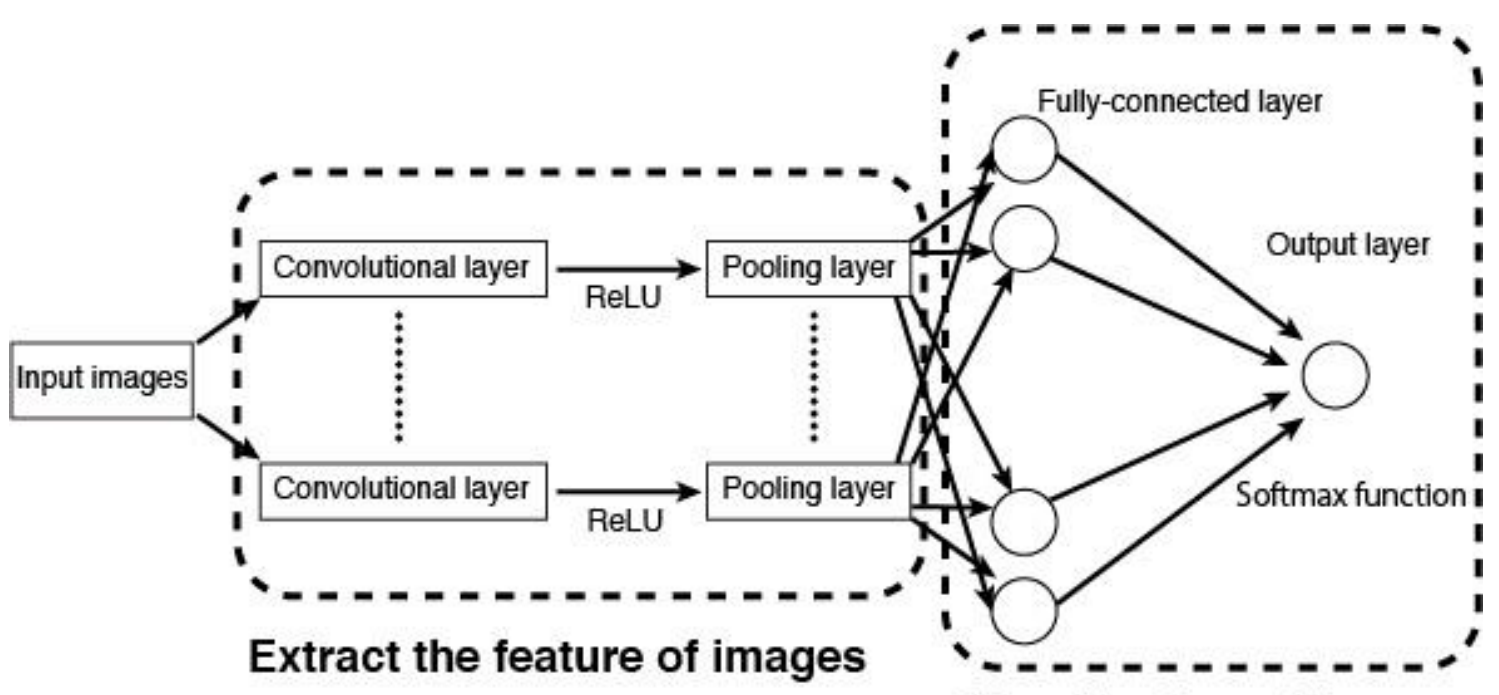

92 FIGURE 2 Schematic diagram of the convolutional neural networks.

93

A feedforward neural network is an artificial neural network wherein connections between the nodes do not form a cycle. These networks, which conduct modeling similar to the neuron activity in the brain, are generally presented as systems of interconnected processing units (artificial neurons) that can compute values from inputs leading to an output that may be used on further units. Artificial neurons are basically processing units that compute some operation over several input variables and, usually, have one output calculated through the activation function. Typically, an artificial neuron has a weight $w_{i}$ that represents the degree of connection between artificial neurons, some input variables $x_{i}$, and a threshold vector $b$. Mathematically, the total input and output of artificial neurons can be described as follows:

106 
109 where $u, z, x, w$, and $b$ represent the total input, output, input variables, weights, and

110 bias, respectively. $f(\cdot)$ denotes an activation function; a nonlinear function such as a

111 sigmoid, hyperbolic, or rectified linear function is provided in $f(\cdot)$. We employed a

112 rectified linear function as the activation function, and this function is referred to as the

113 Rectified Linear Unit (ReLU). The definition of ReLU is shown in the following

114 equation:

$116 f(u)=\max \{0, u\}=\left\{\begin{array}{l}u(u>0) \\ 0(u \leq 0)\end{array}\right.$

As mentioned above, a CNN is a special type of feedforward neural network that

119 is usually used in image classification and identification. A CNN consists of a convolutional layer and pooling layer. The convolutional layer plays a role in capturing the features from the images. In this process, a fixed-sized window runs over the image and extracts the patterns of shades of colors in the image. After each convolutional

123 layer, there are pooling layers that are created in order to reduce the variance of

124 features, which is accomplished by computing some operation of a particular feature over a region of the image.

127 feature that is extracted at the convolution layer so that the output amount of the pooling

128 layer does not change even when the position of the feature amount extracted by the

129 convolution layer is shifted within the image. Two operations may be realized on the 
130 pooling layers, namely, max or average operations, in which the maximum or mean

131 value is selected over the feature region, respectively. This process ensures that the

132 same results can be obtained, even when image features have small translations or

133 rotations, and this is very important for object classification and detection. Thus, the

134 pooling layer is responsible for sampling the output of the convolutional one and

135 preserving the spatial location of the image, as well as selecting the most useful features

136 for the next layers.

137 After several convolutional and pooling layers, there are fully connected ones,

138 which take all neurons in the previous layer and connect them to every single neuron in

139 its layer.

140 Finally, following all of the convolution, pooling, and fully connected layers, a

141 classifier layer may be used to calculate the class probability of each instance. We

142 employed the softmax function in this layer. The softmax function calculates the

143 probabilities of each target class over all possible target classes. The softmax function is

144 written as follows:

145

$146 \quad y_{k}=\operatorname{softmax}_{k}\left(u_{1}, u_{2}, \cdots, u_{K}\right)=\frac{e^{u_{k}}}{\sum_{j=1}^{K} e^{u_{j}}}$

148 where $k$ represents the number of the output unit and $u$ represents input variables.

149 In order to evaluate the performance of the network, a loss function needs to be

150 defined. The loss function evaluates how well the network models the training dataset.

151 The goal of the training is to minimize the error of the loss function. Eq. (5) presents the

152 cross entropy of the softmax function that was employed in this study: 
$154 E=-\sum_{n=1}^{N} \sum_{k=1}^{K} t_{n, k} \log y_{k}$

155

156 where $t$ is the vector for the training data, $K$ represents the possible class, and $N$

157 represents the total number of instances.

158

159 Approach

160 A schematic diagram of our approach is shown in Figure 3. We prepared the training

161 data by using the chopped picture method ${ }^{14}$. First, in this method, we collected the

162 images that were (1) nearly $100 \%$ covered by bamboo and (2) not covered by bamboo.

163 Next, we "chopped" this picture into small squares with $50 \%$ overlap both vertically

164 and horizontally.
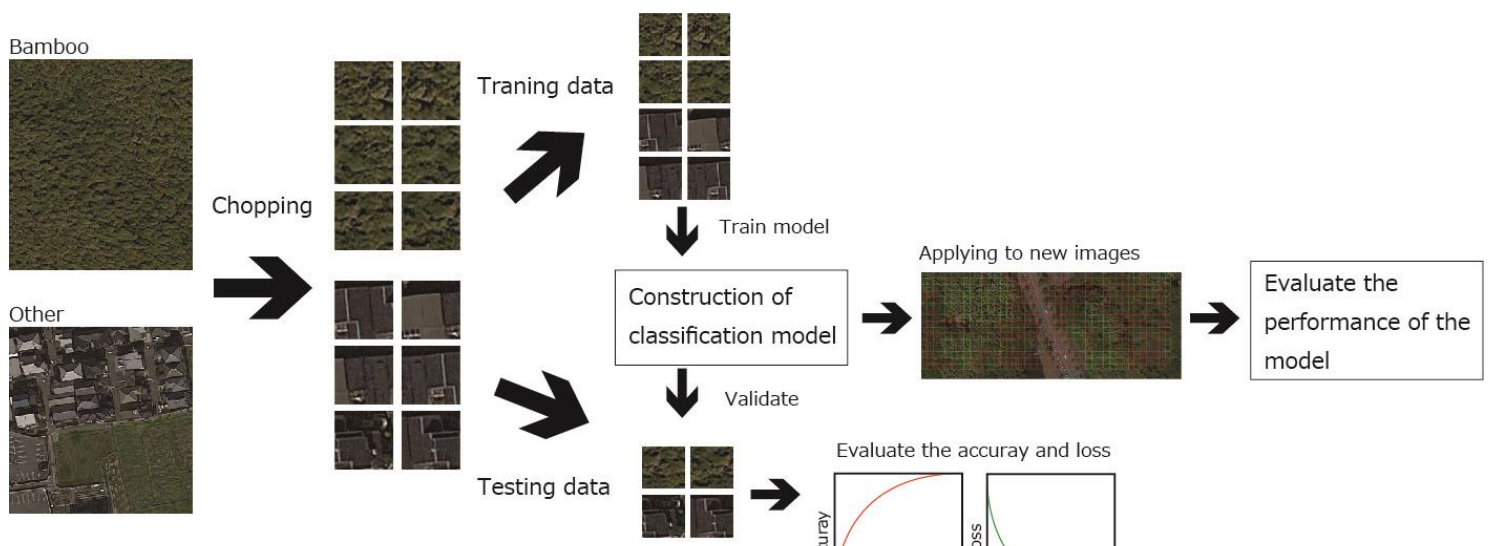

166 FIGURE 3 Schematic diagram of the research approach. This figure was

167 generated using data from Google Earth image (Image data: C2018

168 CNES/Airbus \& Digital Globe). 
171 forest detection. As opposed to traditional approaches of training classifiers with hand-

172 designed feature extraction, the CNN learns the feature hierarchy from pixels to

173 classifiers and trains layers jointly. We used the final layer of the $\mathrm{CNN}$ model for

174 detecting the bamboo coverage from Google Earth images. To make a model for object

175 identification, we used the deep learning framework of NVIDIA DIGITS ${ }^{19}$. We used

$17675 \%$ of the obtained images as training data and the remaining $25 \%$ as validation data.

177 We used the LeNet network model ${ }^{20}$. The model parameters implemented in this study

178 included the number of training epochs (30), learning rate (0.01), train batch size (64),

179 and validation batch size (32).

180

\section{Evaluation of the learning accuracy}

182 Validation of the model in each learning epoch was conducted by using the accuracy

183 and loss function obtained from cross validation images. The accuracy indicates how

184 accurately the model can classify the validation images. Loss represents the inaccuracy

185 of the prediction of the model. If model learning is successful, loss (val) is low and

186 accuracy is high. However, when loss (val) becomes high during learning, this indicates

187 that over fitting is occurring.

189 Evaluation of the model performance

190 We obtained 10 new images, which were uniformly covered by bamboo forest or

191 objects other than bamboo forest, from each study site. Next, we re-sized the images by

192 using the chopped picture method. Third, we randomly sampled 500 images from the 
re-sized images. Finally, we applied the model to the sampled images and evaluated the

194 classification accuracy. To evaluate the performance of the model, we classified the

195 classification results into the following four categories: true positive $(T P)$, false positive

$196(F P)$, false negative $(F N)$, and true negative $(T N)$. Next, we calculated the classification

197 accuracy, recall rate, and precision rate by using the following equations:

198

199

Classification accuracy $=(T P+T N) /(T P+T N+F P+F N)$

202

203

Precision rate $=T P /(T P+F P)$

204

205

\section{Testing the effects of image resolution on the classification accuracy}

To quantify the effects of image resolution on the accuracy of detection, we obtained images at a zoom level of 1/500 ( 0.13 m/pixel spatial resolution), 1/1000 ( 0.26 $\mathrm{m} /$ pixel spatial resolution), and 1/2500 ( 0.65 m/pixel spatial resolution) from each study site. Next, we applied the chopped picture method. To adjust the spatial extent of each chopped image, we chopped 56 pixels for the 1/500 level, 28 pixels for the 1/1000

211 level, and 14 pixels for the 1/2500 level. After constructing the model, we applied the

212 model to the new images and calculated the classification accuracy, recall rate, and

213 precision rate. 
216 To quantify the effects of the spatial extent of the chopping grid on the accuracy of

217 detection, we chopped the 1/500 images at each study site for three types of pixel sizes

$218(84,56,28)$. After constructing the model, we applied the model to new images and

219 calculated the classification accuracy, recall rate, and precision rate.

220

221 Transferability test

222 Given the large amount of variation in the visual appearance of bamboo forest across different cities, it is of interest to study to what extent a model learned on one geographical location can be applied to a different geographical location. As such, we performed experiments in which we trained a model for one (or more) cities, and then, we applied the model to a different set of cities. Performance of the model was evaluated by the classification accuracy, recall rate, and precision rate.

230 Fluctuation of accuracy and loss during the learning epochs

231 The accuracy for classifying the validation data of the final layer ranged from $94 \%$ to

$23299 \%$. Loss values for the validation data ranged from 0.008 to 0.214 (Figure 4). Values

233 of accuracy increased and loss decreased following the learning epochs (Figure 4).

234 These results suggest the all of the models were not overfit to the datasets and

235 successfully learned the features of chopped pictures. 

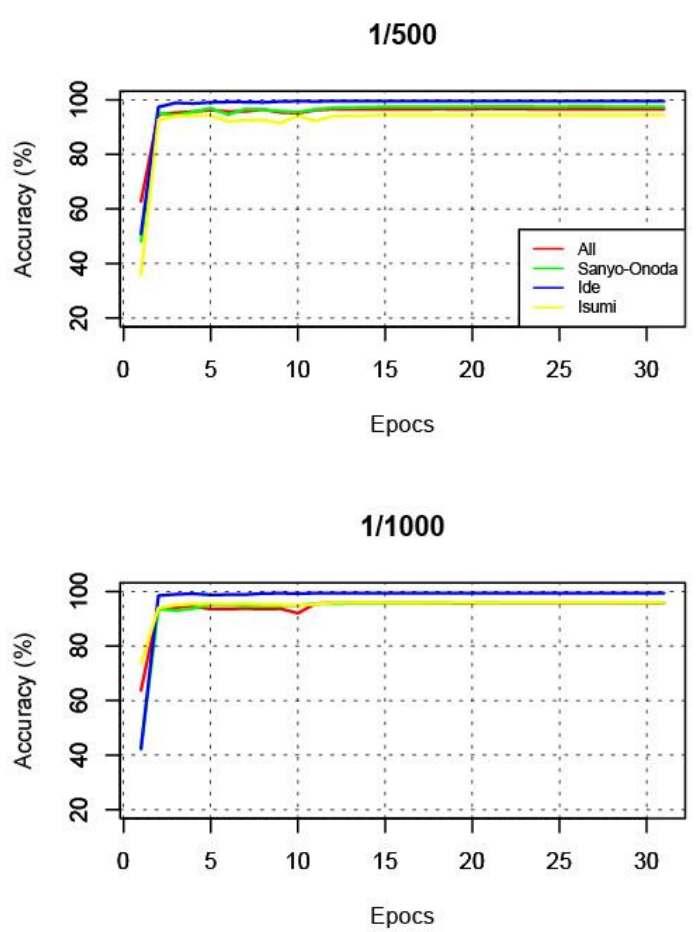

$1 / 2500$

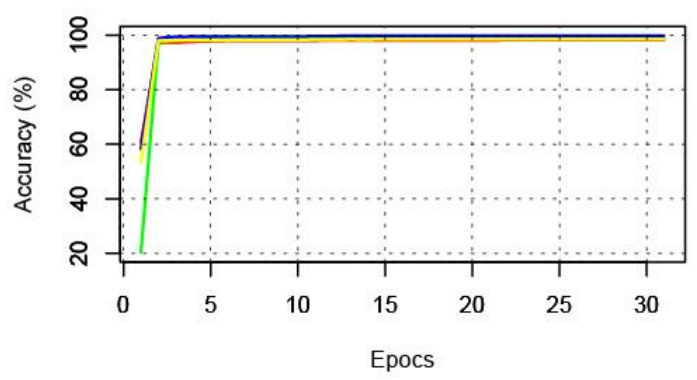

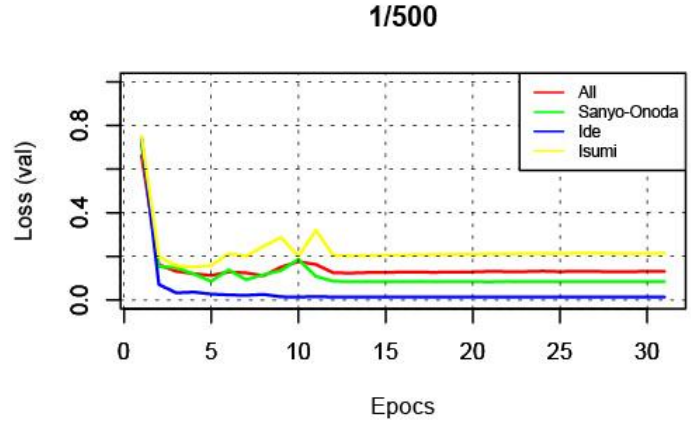
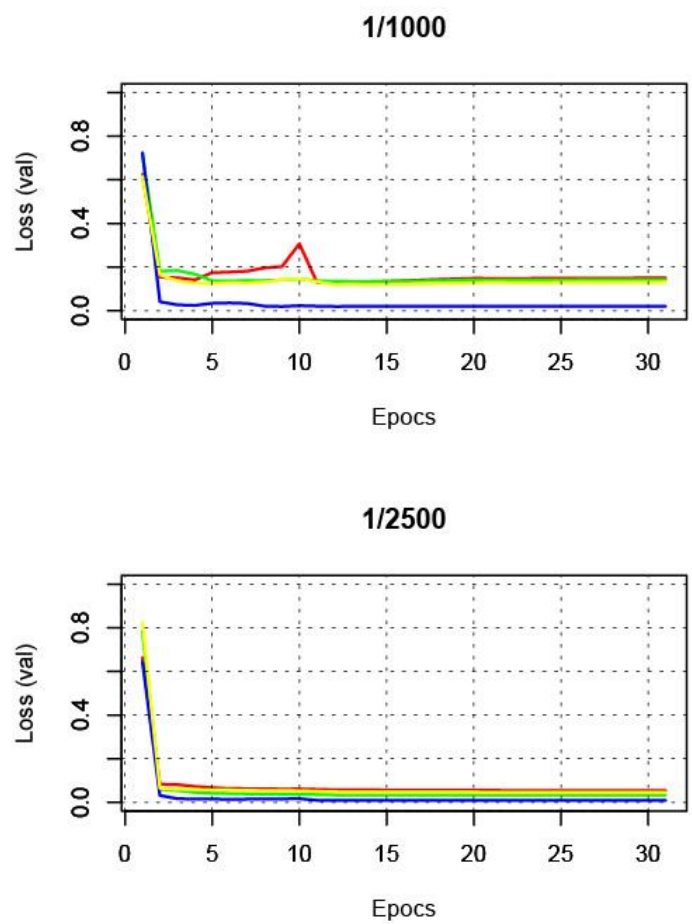

237 FIGURE 4 Accuracy and loss (val) of the model at each learning epoch.

\section{Effects of image resolution on the classification accuracy}

240 The classification accuracy ranged from $76 \%$ to $97 \%$ (Figure 5a). The recall rate and

241 precision rate for bamboo forest ranged $52 \%$ to $96 \%$ and $91 \%$ to $99 \%$, respectively

242 (Figure 5b, d). The recall rate and precision rate for objects other than bamboo forest

243 ranged from $92 \%$ to $99 \%$ and $67 \%$ to $96 \%$, respectively (Figure 5c, e). The recall rate

244 for bamboo forest declined following the decline in the image resolution, and it declined 

$5 a)$.

(a) Classification accuracy

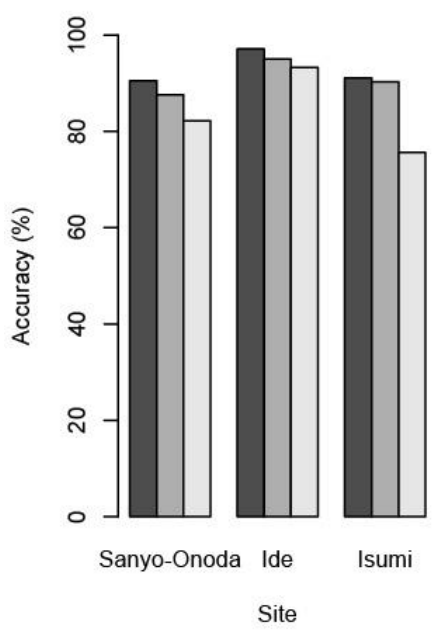

(d) Bamboo forest

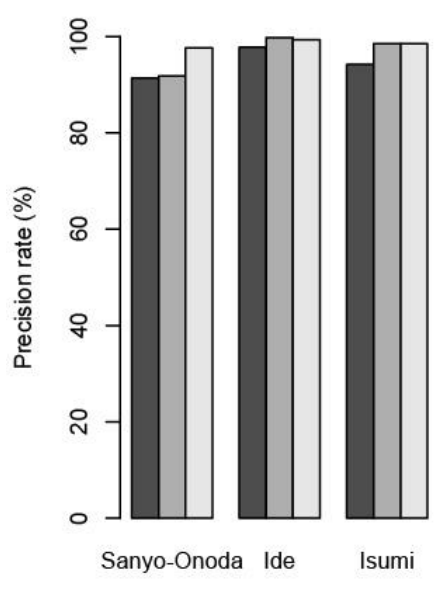

Site (b) Bamboo forest

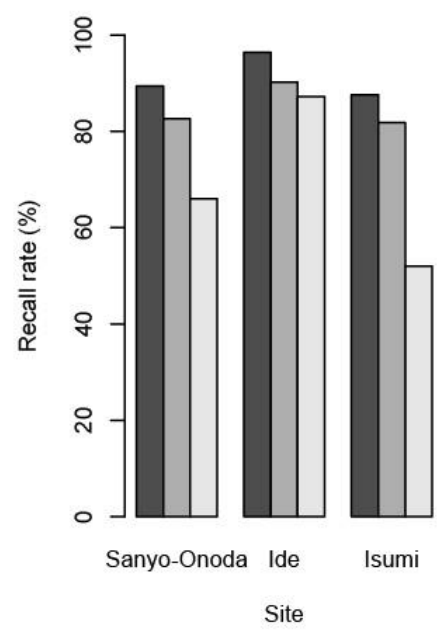

(e) Objects other than bamboo forest

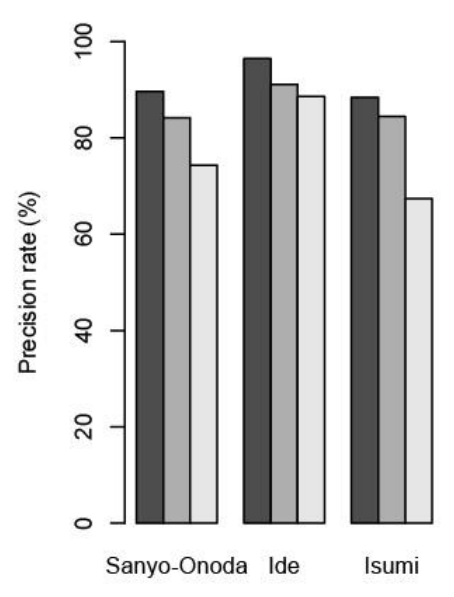

Site (c) Objects other than bamboo forest

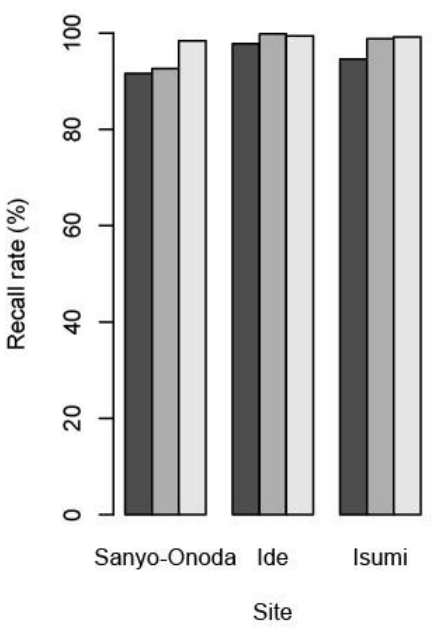

$1 / 500 \square 1 / 1000 \square 1 / 2500$

FIGURE 5 Sensitivity of the image scale versus the test accuracy.

\section{Effects of chopping grid size on the classification accuracy}

251 The classification accuracy ranged from $85 \%$ to $96 \%$ (Figure 6a). The recall rate and

252 precision rate for bamboo forest ranged from $79 \%$ to $99 \%$ and $89 \%$ to $98 \%$, respectively

253 (Figure 6b, d). The recall rate and precision rate for objects other than bamboo forest 
254 ranged from $88 \%$ to $98 \%$ and $79 \%$ to $99 \%$, respectively (Figure $6 \mathrm{c}$, e). The

255 intermediate-sized images (56 pixel) showed the highest classification accuracy at all

256 sites (Figure 6a). An example of a classification image is shown in Figure 7.

(a) Classification accuracy

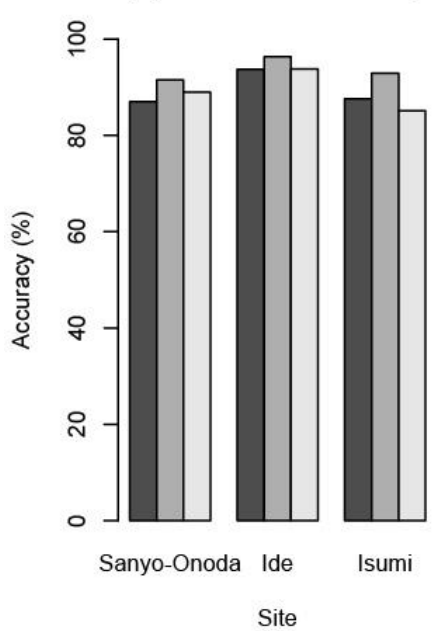

(d) Bamboo forest

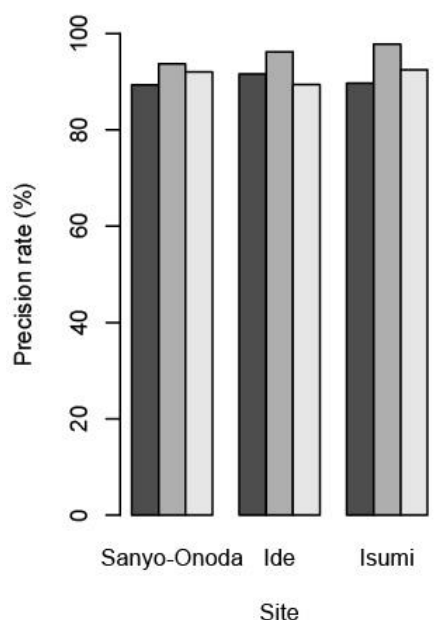

(b) Bamboo forest

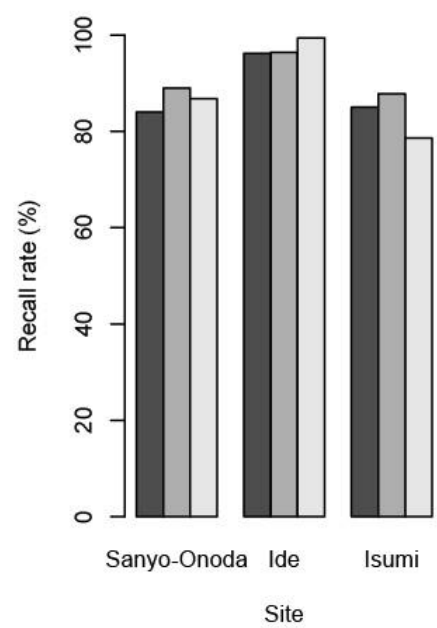

(e) Objects other than bamboo forest

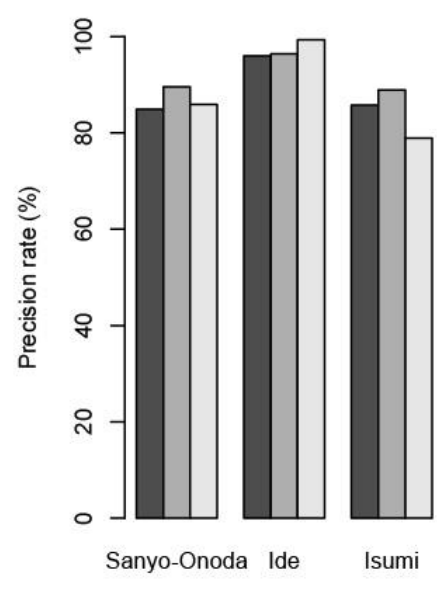

Site (c) Objects other than bamboo forest

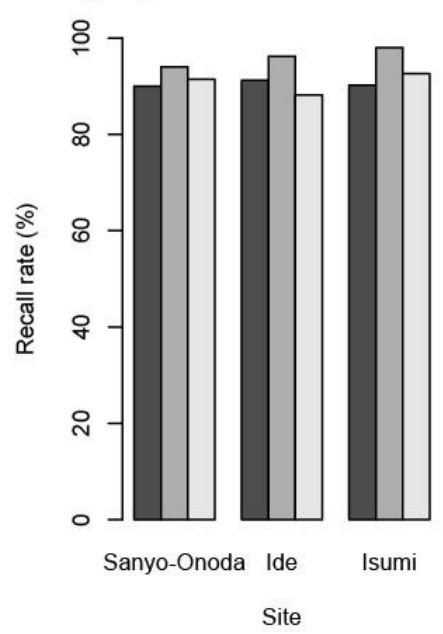

28pix $\square$ 56pix $\square$ 84pix

FIGURE 6 Sensitivity of the pixel size versus the test accuracy. 

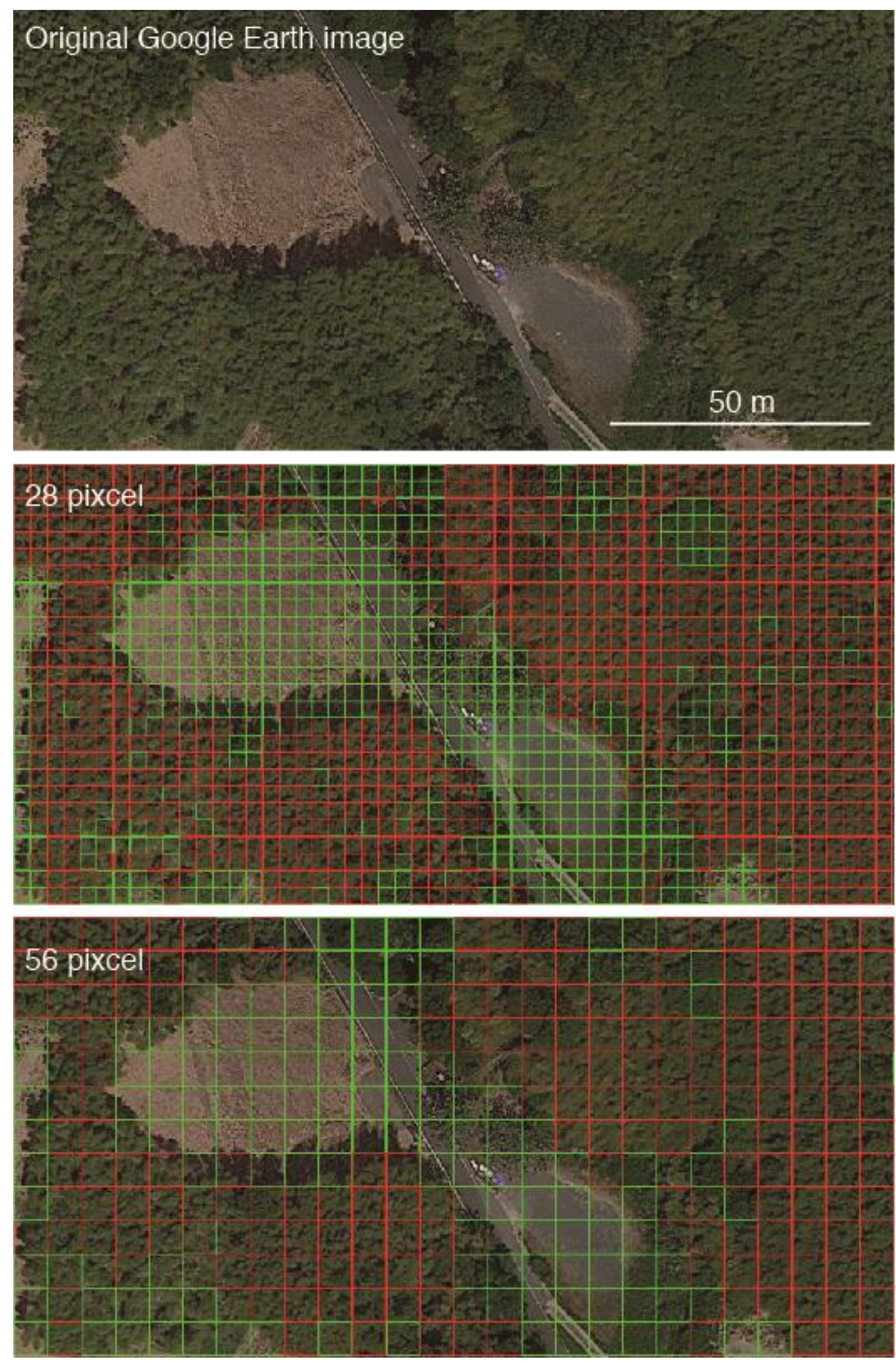

264 FIGURE 7 Example of a classification image. Bamboo forests are highlighted by 
266 generated using data from Google Earth image (Image data: (C2018

267 CNES/Airbus \& Digital Globe).

268

269 Transferability and classification performance

270 In general, performance was poor when training on samples from a given city and

271 testing on samples from a different city (Figure 8a). When the model that was trained by

272 the images of Isumi city was applied to the other cities, the recall rate was the worst

273 (Figure 8b). Conversely, the model that was trained by the images of Sanyo city showed

274 the highest recall rate (Figure 8b). We noticed that a more diverse set (all) did not yield

275 a better performance when applied at different locations than the models trained on

276 individual cities (Figure 8). 

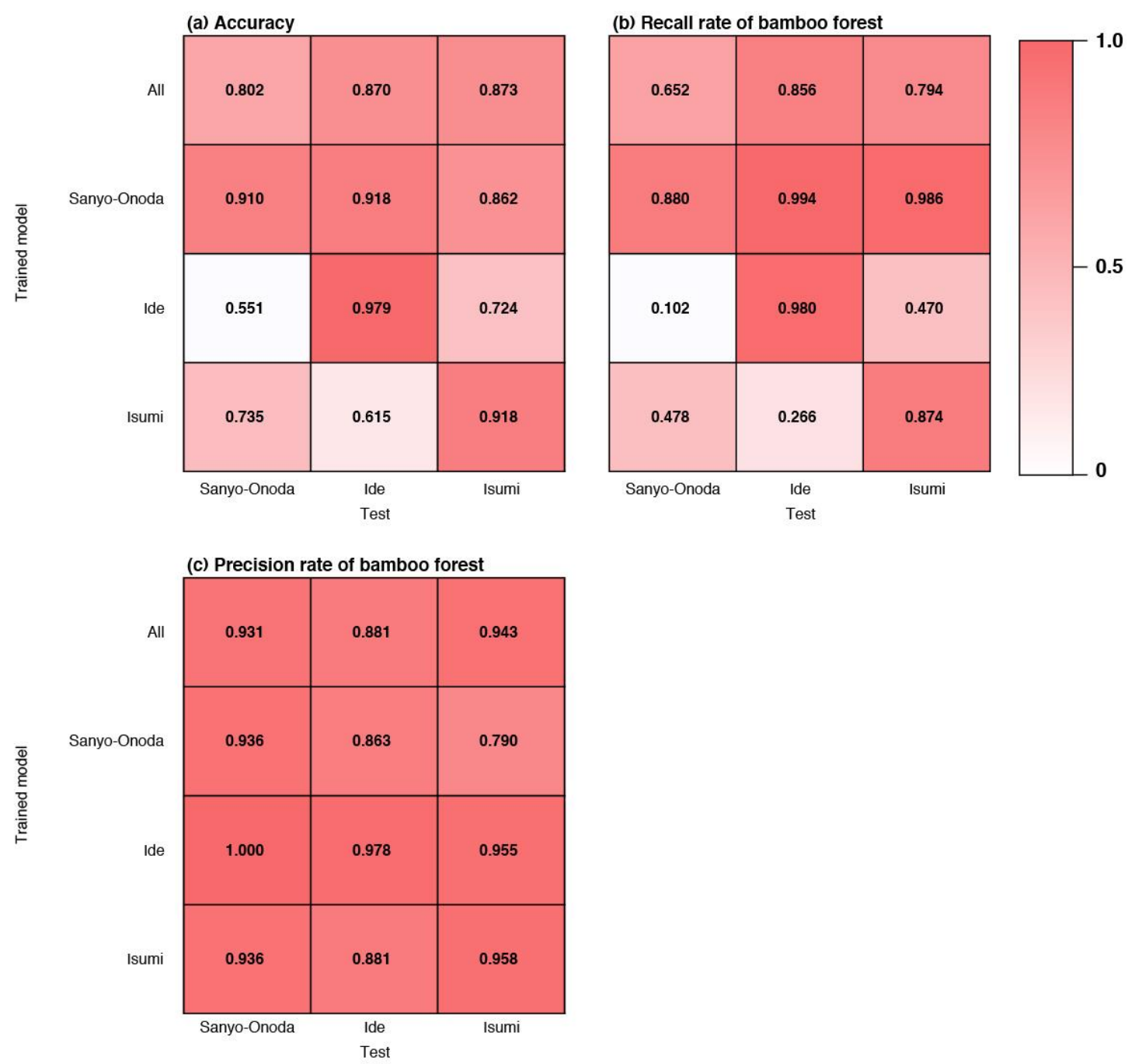

278 FIGURE 8 Transferability of the models learned at one location and applied at another.

\section{DISCUSSION}

282 In this paper, we demonstrated that the chopped picture method and CNN could

283 accurately detect bamboo forest in Google Earth imagery (see Figure 7). Recent

284 research has shown that the deep learning technique is applicable to plant science

285 research ${ }^{8-11,21,22}$; however, applications of DA in plant science have been mainly

286 restricted to photographs taken indoors, and applications to plants in the aerial 
287 photographs are still limited ${ }^{11}$. To the best of our knowledge, this is the first study to

288 successfully identify plant communities automatically from Google Earth imagery.

289 Classifying vegetation from remote sensing images generally suffers from

290 several problems, e.g., it can be difficult to establish a specific pattern for each species,

291 given the high intraclass variance, and also to distinguish between different species,

292 given the interclass similarity of distinct $\operatorname{species}^{12,23}$. So far, it is generally been difficult

293 to detect ambiguous objects such as vegetation, but our results showed good

294 performance during the detection of bamboo forest from Google Earth images by using

295 the chopped picture method even though we employed the most classical CNN (LeNet).

296 Our results highlight that the chopped picture method and CNN would be a powerful

297 method for high accuracy automated bamboo forest detection and vegetation mapping

298 (see Figure 9). 

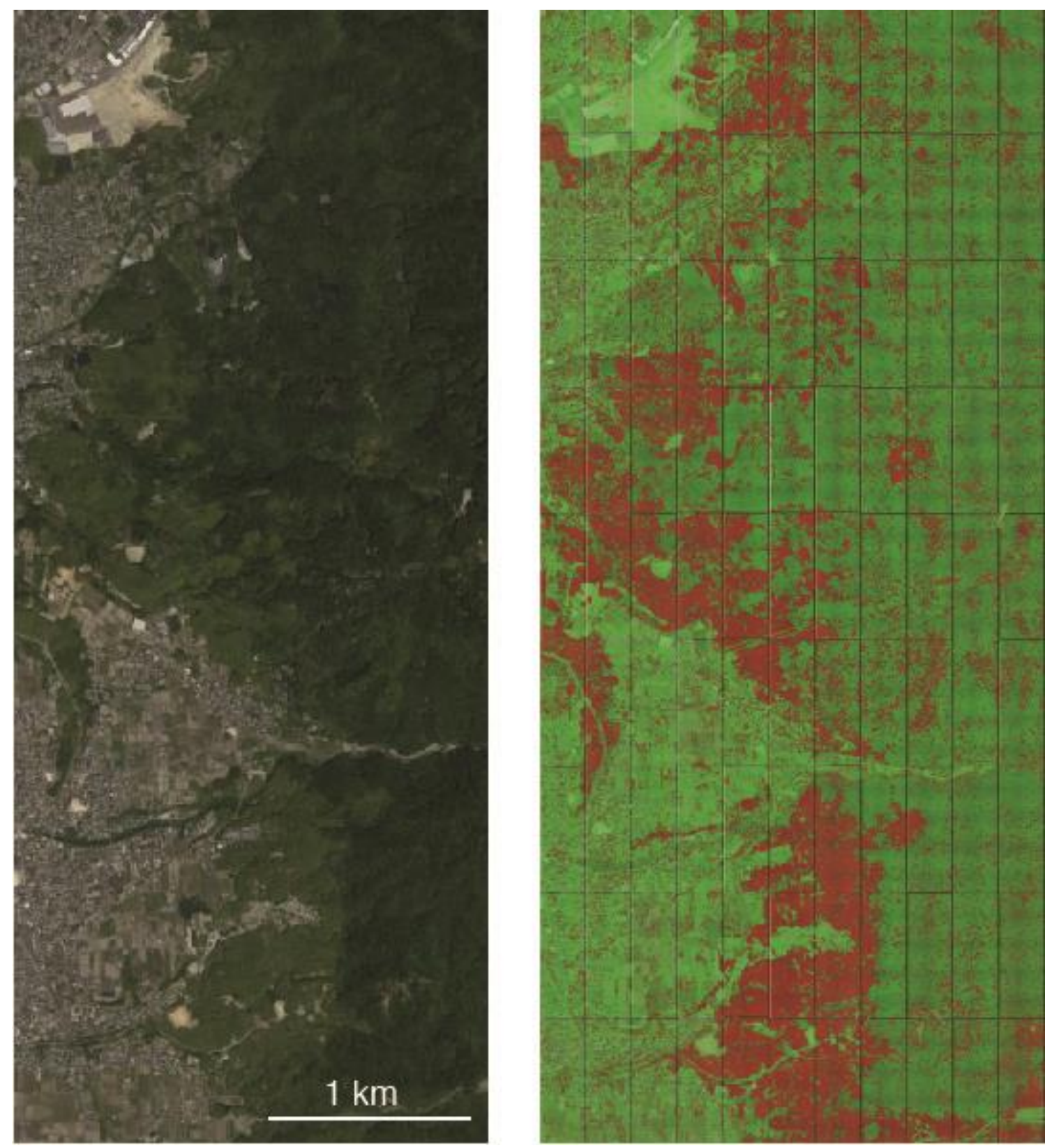

300 FIGURE 9 Example of applying the model to the wide area of Ide city. The left

301 image is the original Google Earth image, and the right image shows the results

302 of bamboo forest detection. Bamboo forests are highlighted by red, and objects

303 other than bamboo are highlighted by green. This figure was generated using

304 data from Google Earth image (Image data: @2018 CNES/Airbus \& Digital

305 Globe). 


\section{Effects of image resolution on the classification accuracy}

309 Our results indicate that the image resolution strongly affects the identification accuracy

310 (Figure 4). As the resolution rate decreased, performance of the model also declined

311 (Figure 4).

312 Especially in the 1/2500 imagery, the recall rate for bamboo forest of Sanyo-

313 Onoda and Isumi city declined to $53 \%$ and 64\%, respectively (Figure $4 \mathrm{~b}$ ). In contrast,

314 the precision rate for bamboo forest increased as the resolution decreased (Figure 4d).

315 This result means that as the resolution decreases, the model overlooks many bamboo

316 forests; thus, when the image resolution is low, it is difficult to learn the features of the

317 object. This result also suggests that in the deep learning model, misidentification due to

318 false negatives is more likely to occur than misidentification due to false positive as the

319 image resolution declines.

321 Effects of chopping grid size on the classification accuracy

322 Our results indicate that the chopping grid size also affects the performance of the

323 model. The classification accuracy was the highest at the medium pixel size $(56 \times 56$

324 pixels; Figure 5a). In contrast to the effects of image resolution, the recall rate and

325 precision rate for bamboo forest was also the highest at the medium pixel size except for

326 the recall rate at Ide city (Figure $5 b, d$ ).

327 This result means that if the grid size is inappropriate, both false positives and false negatives will increase. 
330 chopped pictures in which objects other than bamboo and bamboo are mixed. In this

331 paper, because we evaluated the performance of the model by using images that were

332 uniformly covered by bamboo forest or objects other than bamboo forest, the effects of

333 imagery consisting of mixed objects on the classification accuracy could not be

334 evaluated. Evaluation of the classification accuracy for such images will take place in

335 future research.

\section{Transferability among the models}

338 Results for the transferability tests showed that transferability was generally poor and

339 suggest that the spatial extent of acquisition of training data strongly influences the

340 classification accuracy (Figure 8). The model trained by Sanyo-Onoda city images

341 yielded high recall rates for the images taken at all of the study sites, but the precision

342 rate was lower than that of the other models (Figure 8b, c). This means that the model

343 trained by Sanyo-Onoda city images tends to make false positive mistakes.

344 Interestingly, transferability was not found to be related to the distance among the study

345 sites (Figure 8). This result indicates that classification accuracy across the model

346 reflects the conditions at the local scale such as the climate at the time when the image

347 was taken. Additionally, even when we applied a model that learned from all training

348 images (all), the performance of the model was not as good as when the training data

349 were obtained within the same city. The same tendencies have been reported in studies

350 that classified land use by using deep learning ${ }^{24}$. This may suggest that increasing the

351 number of training data may also lead to a decrease in the identification accuracy, and it

352 may be difficult to construct an identification model applicable to a broad area. 


\section{Conclusions and future directions}

355 Our results show that the deep learning model presented here can detect bamboo forest

356 from Google Earth images accurately. Our results also suggest that deep learning and

357 the chopped picture method would be a powerful tool for high accuracy automated

358 vegetation mapping and may offer great potential for reducing the effort and costs

359 required for vegetation mapping as well as improving the current status of monitoring

360 the distribution of bamboo. Recently, bamboo expansion has become an important

361 problem in Japan because of its invasiveness ${ }^{17}$. While some research has analyzed the

362 bamboo forest distribution probability on a national scale ${ }^{25,26}$, monitoring of bamboo

363 expansion is still a challenging problem because of labor requirements. Our approach

364 could potentially lead to the creation of a semi or even fully automated system for the

365 monitoring of bamboo expansion.

366 Our results also suggest that the identification accuracy depends on the image

367 resolution and chopping grid size. Especially, the spatial resolution of training data

368 strongly affects the model performance. Generally, satellite-based remote sensing has

369 been widely studied and applied but suffers from insufficient information due to low

370 resolution images or inaccurate information due to local weather conditions ${ }^{27}$. Our

371 results also show that the performance of the model can be greatly influenced by the

372 spatial extent of the acquired training data and a model learned on one geographical

373 location is difficult to apply to a different geographical location. It remains a future task

374 to develop a model that can be applied over a wide spatial scale. 
bioRxiv preprint doi: https://doi.org/10.1101/351643; this version posted November 7, 2018. The copyright holder for this preprint (which

was not certified by peer review) is the author/funder. All rights reserved. No reuse allowed without permission.

377 This work was supported by JST PRESTO, Japan (Grant No. JPMJPR15O1).

378 


\section{Author Contributions}

380 S. W designed the research based on discussion with T. I. S. W and K. S

381 conducted research. S. W and T. I wrote the manuscript.

382

\section{Competing interests}

384 The authors declare no competing interests.

385

386

387 


\section{REFERENCES}

389 1. Franklin, J. Mapping Species Distributions: Spatial Inference and Prediction.

$390 \quad$ (Cambridge University Press, 2009).

2. Xie, Y., Sha, Z., \& Yu, M. Remote sensing imagery in vegetation mapping: a review. Journal of Plant Ecology, 1, 9-23. doi: 10.1093/jpe/rtm005 (2008).

3. Hearst, M. A., Dumais, S. T., Osuna, E., Platt, J., \& Schölkopf, B. Support vector machines. IEEE Intelligent Systems and their Applications, 13, 18-28. doi: 10.1109/5254. 708428 (1998).

4. Bengio, Y. Learning deep architectures for AI. Foundations and Trends in Machine Learning, 2, 1-127 (2009).

5. Goodfellow, I., Bengio, Y. \& Courville, A. Deep Learning (MIT press, 2016)

6. Karpathy, A., Toderici, G., Shetty, S., Leung, T., Sukthankar, R., \& Fei-Fei, L. Large-scale video classification with convolutional neural networks. Proceedings of the IEEE Conference on Computer Vision and Pattern Recognition (Columbus), 1725-1732 (2014).

7. Yosinski, J., Clune, J., Bengio, Y., \& Lipson, H. How transferable are features in deep neural networks? Advances in Neural Information Processing Systems (Montreal), 3320-3328 (2014).

8. Mohanty, S. P., Hughes, D. P., \& Salathé, M. Using deep learning for image-based plant disease detection. Frontiers in Plant Science, 7, 1419. doi: 10.3389/fpls.2016.01419 (2016).

9. Ramcharan, A., Baranowski, K., McCloskey, P., Ahamed, B., Legg, J., \& Hughes, 
412 10. Carranza-Rojas, J., Goeau, H., Bonnet, P., Mata-Montero, E., \& Joly, A. Going

413 deeper in the automated identification of Herbarium specimens. BMC Evolutionary

$414 \quad$ Biology, 17, 181 (2017).

415 11. Nogueira, K., Penatti, O. A. B., \& Dos Santos, J. A. Towards better exploiting

416 convolutional neural networks for remote sensing scene classification. Pattern

$417 \quad$ Recognition, 61, 539-556 (2017).

418 12. Zhu, X.X., Tuia, D., Mou, L., Xia, G.S., Zhang, L., \& Xu, F. Deep Learning in

419 Remote Sensing: A Comprehensive Review and List of Resources. IEEE Geosci.

$420 \quad$ Remote Sens. Mag, 5, 8-36 (2017).

421 13. Guirado, E., Tabik, S., Alcaraz-Segura, D., Cabello, J., \& Herrera, F. Deep-learning

422 Versus OBIA for Scattered Shrub Detection with Google Earth Imagery: Ziziphus

423 lotus as Case Study. Remote Sensing, 9, 1220. doi: 10.3390/rs9121220 (2017).

424 14. Ise, T., Minagawa, M., \& Onishi, M. Classifying 3 moss species by deep learning, 425 using the "chopped picture" method. Open Journal of Ecology, 8, 166-173 (2018).

426 15. Nakashima, A. The present situation of the bamboo forests management in the 427 suburbs: A case study of the bamboo shoot producing districts in the suburbs of 428 Kyoto City. Applied Forest Science, 10, 1-7 (in Japanese with English abstract) $429 \quad$ (2001).

430 16. Nishikawa, R., Murakami, T., Yoshida, S., Mitsuda, Y., Nagashima, K., \& Mizoue, $431 \quad$ N. Characteristic of temporal range shifts of bamboo stands according to adjacent 432 landcover type. Journal of the Japanese Forestry Society, 87, 402-409 (in Japanese 433 with English abstract) (2005). 
434 17. Okutomi, K., Shinoda, S., \& Fukuda, H. Causal analysis of the invasion of broad-

435 leaved forest by bamboo in Japan. Journal of Vegetation Science, 7, 723-728

$436 \quad$ (1996).

437 18. Suzuki, S. Chronological location analyses of giant bamboo (Phyllostachys

438 pubescens) groves and their invasive expansion in a satoyama landscape area,

439 western Japan. Plant Species Biology, 30, 63-71 (2015).

440 19. NVIDIA NVIDIA deep learning gpu training system.

441 https://developer.nvidia.com/digits. Accessed June 1, 2018 (2016).

442 20. LeCun, Y. L., Bottou, L., Bengio, Y., \& Haffner, P. Gradient-based learning applied 443 to document recognition. Proceedings of the IEEE, 86, 2278-2324. doi:

$444 \quad 10.1109 / 5.726791(1998)$.

445 21. Wäldchen, J., Rzanny, M., Seeland, M., \& Mäder, P. Automated plant species

446 identification - Trends and future directions. PLoS Computational Biology, 14(4),

447 e1005993. https://doi.org/10.1371/journal.pcbi.1005993 (2018).

448 22. Wäldchen, J., \& Mäder, P. Machine learning for image based species identification.

$449 \quad$ Methods Ecol Evol. 2018, 1-10. doi: 10.1111/2041-210X.13075 (2018).

450 23. Rocchini, D., Foody, G. M., Nagendra, H. et al. Uncertainty in ecosystem mapping

451 by remote sensing. Computers and Geosciences, 50, 128-135 (2013).

452 24. Albert, A., Kaur, J., \& Gonzalez, M. Using convolutional networks and satellite

453 imagery to identify patterns in urban environments at a large scale. arXiv preprint, $454 \quad$ arXiv:1704.02965 (2017).

455 25. Someya, T., Takemura, S., Miyamoto, S., \& Kamada, M. Predictions of bamboo 456 forest distribution and associated environmental factors using natural environmental 
26. Takano, K. T., Hibino, K., Numata, A., Oguro, M., Aiba, M., Shiogama, H.,

460 Takayasu, I., \& Nakashizuka, T. Detecting latitudinal and altitudinal expansion of invasive bamboo Phyllostachys edulis and Phyllostachys bambusoides (Poaceae) in Japan to project potential habitats under $1.5^{\circ} \mathrm{C}-4.0^{\circ} \mathrm{C}$ global warming. Ecology and Evolution, 7, 9848-9859 (2017).

464 27. Jones, H. G., \& Vaughan, R. A. Remote Sensing of Vegetation: Principles

465 Techniques and Applications (Oxford University Press, 2010).

466

467

468

469

470

471

472

473

474 